\begin{tabular}{|c|c|}
\hline Title & $\begin{array}{l}\text { Nonequilibrium Green's function study on the electronic structure and transportation behavior of the conjugated } \\
\text { molecular junction : Terminal connections and intramolecular connections }\end{array}$ \\
\hline Author(s) & $\begin{array}{l}\text { Liu, Hongmei; Ni, Wenbin; Zhao, Jianwei; Wang, Nan; Guo, Y an; Taketsugu, Tetsuya; Kiguchi, Manabu; Murakoshi, } \\
\text { Kei }\end{array}$ \\
\hline Citation & $\begin{array}{l}\text { Journal of Chemical Physics, 130(24), } 244501 \\
\text { https://doi.org/10.1063/1.3151682 }\end{array}$ \\
\hline Issue Date & $2009-06-28$ \\
\hline Doc URL & http:/hdl. handle.net/2115/38832 \\
\hline Rights & $\begin{array}{l}\text { Copyright } 2009 \text { A merican Institute of Physics. This article may be downloaded for personal use only. A ny other use } \\
\text { requires prior permission of the author and the A merican Institute of Physics. }\end{array}$ \\
\hline Type & article \\
\hline File Information & 130-24_244501.pdf \\
\hline
\end{tabular}

Instructions for use 


\title{
Nonequilibrium Green's function study on the electronic structure and transportation behavior of the conjugated molecular junction: Terminal connections and intramolecular connections
}

\author{
Hongmei Liu, ${ }^{1}$ Wenbin Ni, ${ }^{1}$ Jianwei Zhao, ${ }^{1, a)}$ Nan Wang,,${ }^{1}$ Yan Guo, ${ }^{1}$ Tetsuya Taketsugu, ${ }^{2}$ \\ Manabu Kiguchi, ${ }^{2}$ and Kei Murakoshi ${ }^{2}$ \\ ${ }^{1}$ Key Laboratory of Analytical Chemistry for Life Science, Ministry of Education, School of Chemistry \\ and Chemical Engineering, Nanjing University, Nanjing 210008, People's Republic of China \\ ${ }^{2}$ Division of Chemistry, Graduate School of Science, Hokkaido University, Sapporo 060-0810, Japan
}

(Received 16 January 2009; accepted 18 May 2009; published online 25 June 2009)

\begin{abstract}
In the recent density functional-based calculations, it was found that the conductivity of naphthalene molecular wires can be modulated by altering the linking position of the molecule to the electrode [D. Walter, D. Neuhauser, and R. Baer, Chem. Phys. 299, 139 (2004)]. A quantum interference model was proposed to interpret the observation. In this paper, we further studied the conductance of a series of conjugated molecules containing aromatic rings using density functional theory combined with nonequilibrium Green's function method. For polyacene systems with different terminal connections, the conductivity is dependent on the substitution position of anchoring groups even with similar electron transport distance. The conductance of trans-substitution can be ten times or more as large as that of the cis-substitution. However, for the biphenyl system with different intramolecular connections, adding more connections between two benzene rings does not change the junction conductance. All these results indicate that the junction conductance is strongly dependent on the particular electron transport pathway. The alternating double-single linkage is the most probable one, since others are impeded by the single bonds. (C) 2009 American Institute of Physics.
\end{abstract}

[DOI: $10.1063 / 1.3151682]$

\section{INTRODUCTION}

Building an electronic device using individual molecules is one of the ultimate goals in electronics. To achieve this, understanding the dependence of transportation on the molecular structure is an important step. ${ }^{1}$

Tremendous experimental efforts have been made to understand electron transfer through a molecule. Some effective techniques have been developed under this heading, for instance, the mechanically controllable break junction, ${ }^{2}$ scanning tunneling microscopy, ${ }^{3}$ conducting atomic force microscopy, ${ }^{4}$ crossed wires, ${ }^{5}$ and mercury drop contacts. ${ }^{6}$ Alternatively, theoretical simulations may also provide insight into the nature of molecular electron transfer. ${ }^{7}$ The first principles calculation together with nonequilibrium Green's function (NEGF) method gives self-consistent treatment of the transportation of the molecular junction, ${ }^{8}$ which has been successfully used to compare with many experimental results. ${ }^{9}$

Both experimental and theoretical investigations inferred that many factors affect the transportation behavior of a molecular junction. Among them, the intrinsic property of the molecules,${ }^{10}$ including their length, ${ }^{10,11}$ conformation, ${ }^{12}$ the charged state, ${ }^{13}$ the gap between the highest occupied molecular orbital (HOMO) and the lowest unoccupied molecular orbital (LUMO) ${ }^{10}$ and the alignment of the energy gap to

\footnotetext{
${ }^{a)}$ Author to whom correspondence should be addressed. Electronic mail: zhaojw@nju.edu.cn.
}

the metal Fermi level, ${ }^{14}$ interaction from the neighboring molecules, ${ }^{15}$ and anchoring group ${ }^{16}$ received special attention. The particular electron transfer pathway has been considered recently. Walter et al. ${ }^{17}$ and Maiti ${ }^{18}$ found that the conductivity of polycyclic hydrocarbon molecule is strongly dependent on the linking position between molecule and electrode. The specific electron transport pathway was also observed in the circular conjugated systems. ${ }^{19,20}$

The circular conjugated molecules are promising materials in nanoelectronics. According to the ring size, the circular conjugated molecules can be classified into three catalogs. The macrocycle, such as phenylacetylene ${ }^{21}$ and diethynylbenzene macrocycle, ${ }^{22}$ has a vacant cavity larger than $1.0 \mathrm{~nm}$ that can bind a molecule or an atomic cluster. The middle conjugated cycle, such as porphyrin, has a smaller cavity, where only a metallic cation can be trapped. ${ }^{19}$ Most small conjugated cycles are composed of benzene ${ }^{23}$ or five-membered rings, ${ }^{24}$ which have high charge mobility and are particularly promising in organic field-effect transistors. ${ }^{24,25}$

Recently, the quantum interference of electron is used to interpret the specific electron transport behavior in the conjugated molecules. ${ }^{17,26-29}$ Baer and Neuhsuser ${ }^{27}$ proposed that quantum interference, i.e., constructive or destructive interference can tune the molecular conductance. They used the simple Hückel model as well as more accurate calculations, such as density functional theory (DFT) to describe this effect. Walter et al. ${ }^{17}$ designed a series of aromatic mol- 


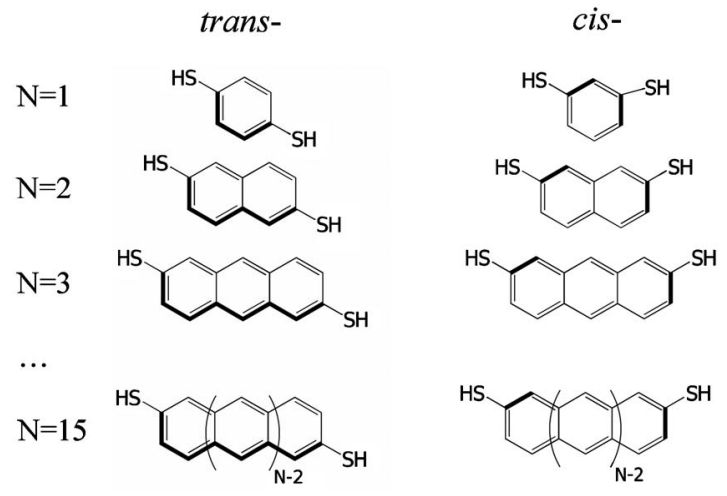

SCHEME 1. The molecular structures of polyacene system with different terminal connections. The substitution of dithiol groups on the same side with respect to the long molecular axis is defined as cis-substitution and on the two sides is noted as trans-substitution. The thick bonds denote the most probable electron transport pathway in trans- $N$, while the thick bonds impede electron transport in $c i s-N$.

ecules and confirmed that the electron transmission is strongly affected by the quantum interference controlled by the molecule-electrode connection. Thus, trans-substituted naphthalene is more conductive than the cis-substituted one. Subsequently, Cardamone et al. ${ }^{28}$ exploited the quantum interference transistor based on the monocyclic aromatic [18] annulenes, in which current was controlled by perfect destructive interference. Ke et $_{\text {al. }}{ }^{29}$ also found the similar effect in the same system. Although the quantum interference is successful in interpreting some molecular electron transport behaviors, it still requires more systems to validate this model.

In this work, we focus on the electron transport pathway in two conjugated systems. The first is polyacene system with different terminal connections as shown in Scheme 1. The substitution of dithiol groups on the same side with respect to the molecular long axis is defined as cis-substitution and that on the opposite side as trans-substitution. For benzene dithiol, the para-substitution is represented as trans-1 and the meta-substitution as cis-1, respectively. The second is BP- $N(N=1-8)$ system based on planar 4,4'-biphenyl dithiol with different intramolecular connections, in which various linkages are used between the two benzene rings (Scheme 2). For all models, the molecule is anchored to a gold surface via a S-Au bond. We also found that the

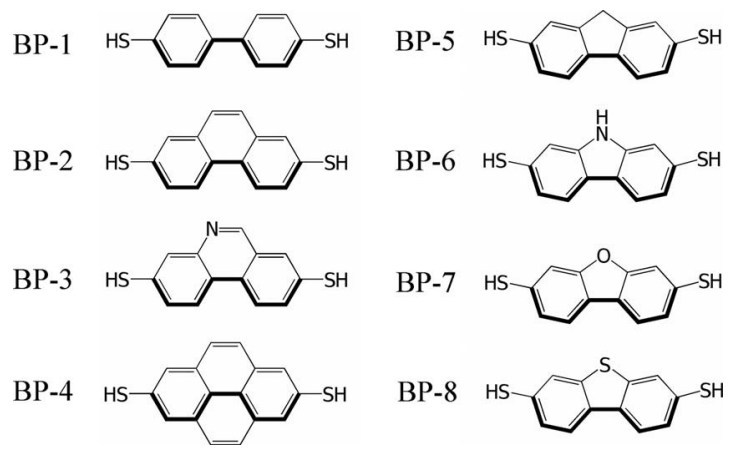

SCHEME 2. The molecular structures of the BP- $N$ system with different intramolecular connections. The thick bonds show the most probable electron transport pathway, which remains the same for different molecules. trans- $N$ and $c i s-N$ series have significantly different conductivities, which is consistent with the DFT-based calculation of polyacene. ${ }^{17}$ Unexpectedly, the BP- $N$ series have similar conductivity though different linkages in the molecules. These results infer that the particular electron transport pathway exists in the molecular junction.

\section{COMPUTATIONAL METHOD}

\section{A. Geometric optimization}

The geometries of the conjugated molecules considered in our calculations were optimized by GAUSSIAN 03 (Ref. 30) at the B3LYP hybrid exchange-correlation functional with the LANL2DZ basis set. In each model, the molecule was sandwiched between two equilateral triangles of $\mathrm{Au}$ atoms, with an $\mathrm{Au}-\mathrm{Au}$ bond length of $2.88 \AA \AA^{10,31,32}$ The thiol lost a hydrogen atom when it attached to the gold triangle, making a strong covalent bond with $\mathrm{Au}$ atoms, providing the good electrical contact between the organic molecule and the metal electrode. In our calculation, we considered sulfur atom chemisorbed at the hollow site of the Au cluster, which is energetically favorable. ${ }^{33}$ The relative positions of $\mathrm{Au}$ atoms were frozen in each triangle, but the distances between the two clusters were relaxed during geometric optimization.

\section{B. Current calculation}

The ATOMISTIX TOOLKIT package ${ }^{8,34}$ was employed in the present work to study the transport behavior of molecular junctions, which combines the well-tested electronic structure calculation by DFT and NEGF technologies. We used a $(3 \times 3)$ unit cell with periodic boundary conditions of the $\mathrm{Au}(111)$ surface to simulate the metallic electrode. ${ }^{32}$ The semi-infinite Au-molecule-Au system was divided into three parts: left electrode, right electrode, and a central scattering region, which contained the molecule and parts of Au layers for fully accommodating the molecule-electrode interaction.

The central scattering region was determined as follows. Each of the optimized molecule was positioned in the hollow site of the $\mathrm{Au}(111)$ surface. After positioning the molecule perpendicularly to the $\mathrm{Au}$ surface, the favorable moleculeelectrode contact distance was fixed at $2.0 \AA$ within the computational accuracy used by most researchers. ${ }^{10,31,32,35}$ For DFT electronic structure calculation, we used the TroullierMartins nonlocal pseudopotentials for core electrons, ${ }^{36}$ double- $\zeta$ plus polarization basis set for the organic molecules, and single- $\zeta$ plus polarization basis set for the $\mathrm{Au}$ electrodes. The local density approximation was used for the electron exchange and correlation.

\section{RESULTS AND DISCUSSION}

\section{A. Terminal connection to the molecular bridge: Different electron transport pathways}

The polyacene systems include one or more benzene rings that form the main body of electron transport pathway. The difference between trans- $N$ and $c i s-N(N=1-15)$ is the substitution position of the thiol groups. For the trans- $N$ series, two thiol groups are substituted on opposite sides, with respect to the long axis of the wire, forming a trans-like 


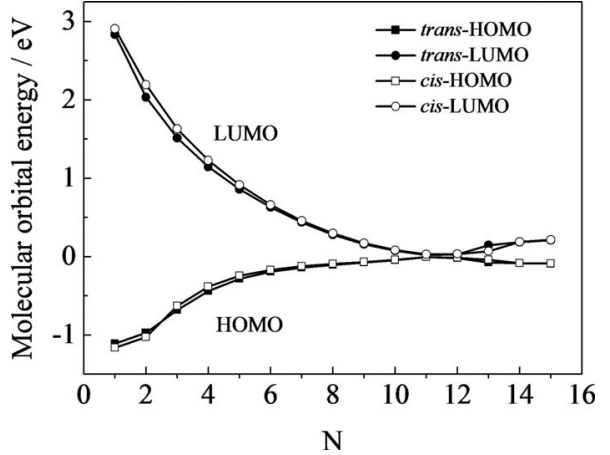

FIG. 1. The HOMO and LUMO energy levels of trans- $N$ and $c i s-N$ models at zero bias.

structure, while on the same side for the $c i s-N$ series. In the theoretical calculation about polyacene by Walter et al. ${ }^{17}$ and Maiti, ${ }^{18}$ the electron quantum interference occurs in the aromatic molecules. For the trans- and cis-configurations, electron transport takes two pathways. The phase difference of the two pathways in cis-configuration is $\pi$. Therefore, the two pathways interfere destructively and the transmission probability is quite low. However, in the trans-configuration, the electron takes two pathways with the same length. Thus, the two pathways interfere constructively and the conductance of trans- $N$ is expected to be high. ${ }^{17}$ Although the quantum interference is intuitively applicable, other microscopic features of the molecular junction should be also elaborated for understanding the electron transfer.

Since many factors, such as energy barrier height [HOMO-LUMO gap, (HLG)], spatial distribution of the frontier molecular orbitals, and transmission spectra, may contribute to the conductance of the molecular junctions, we analyzed the molecule energy level first. Figure 1 and Table I show the HOMO and LUMO energy levels and HLG of those models at zero bias. Although the HLG decreases with increasing molecular length (similar to other conjugated molecules), ${ }^{10}$ the HLG difference between trans- $N$ and $c i s-N$

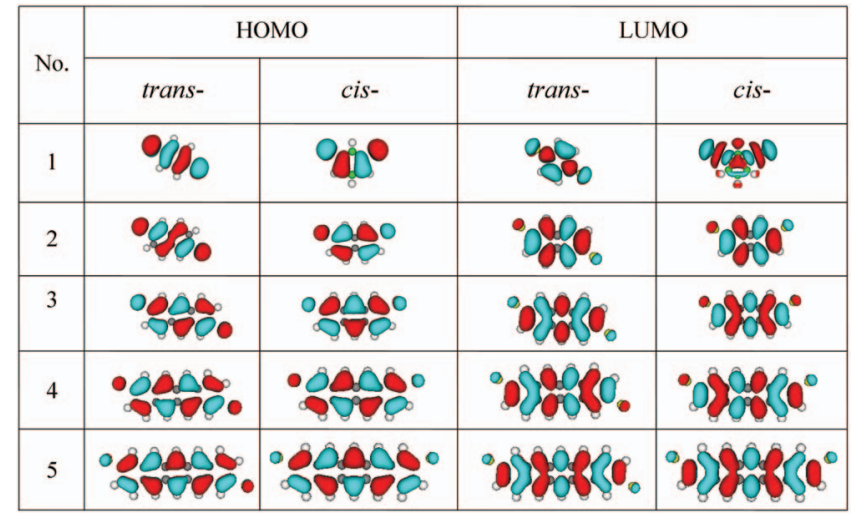

FIG. 2. (Color) The HOMO and LUMO spatial distributions of trans- $N$ and cis- $N$ models.

is not prominent. For example, the HLG of trans-1 $(3.95 \mathrm{eV})$ is only $0.12 \mathrm{eV}$ lower than that of $c i s-1(4.07 \mathrm{eV})$. When the chain length increases, the HLG between trans- and cisbecomes closer as shown in Table I. As the repeat unit number reaches 7, they have the same HLG.

Another static feature of the molecular junction is the spatial distribution that partially represents the mobility of the $\pi$-electron in the molecular system. Figure 2 displays the spatial distribution of the frontier molecular orbitals for models $N=1-5$. Although there is an obvious difference between trans-1 and cis-1 in either HOMO or LUMO, the difference gradually vanishes as the chain length increases. This observation indicates that the main body of electron transport in the long molecular wire is exactly the same in spite of the various terminal substitutions. Despite the similarity of these static properties, electron transport is quite different due to the substitutions, especially for the short molecular chain. This character stimulates us to consider the dynamic transportation of the two connections.

The zero-bias conductance of all models is given in Table I, in which the conductance of model trans-1

TABLE I. Molecular conductance, molecular length, and HLG under zero bias of trans- $N$ and $c i s-N(N$ $=1-15$ ) series studied in the present work.

\begin{tabular}{|c|c|c|c|c|c|c|}
\hline \multirow[b]{2}{*}{ No. } & \multicolumn{2}{|c|}{ Conductance/ $\mu \mathrm{S}$} & \multicolumn{2}{|c|}{ Length/Å (S-M-S) } & \multicolumn{2}{|c|}{ HLG/eV } \\
\hline & trans- & cis- & trans- & cis- & trans- & cis- \\
\hline$N=1$ & 28.5 & 2.4 & 6.52 & 5.78 & 3.95 & 4.07 \\
\hline$N=2$ & 17.5 & 0.5 & 8.76 & 8.26 & 3.01 & 3.22 \\
\hline$N=3$ & 13.1 & 0.2 & 11.11 & 10.74 & 2.19 & 2.27 \\
\hline$N=4$ & 11.8 & 0.7 & 13.50 & 13.23 & 1.59 & 1.61 \\
\hline$N=5$ & 12.3 & 1.7 & 15.92 & 15.71 & 1.14 & 1.16 \\
\hline$N=6$ & 13.6 & 2.7 & 18.36 & 18.19 & 0.82 & 0.83 \\
\hline$N=7$ & 15.5 & 3.0 & 20.80 & 20.69 & 0.58 & 0.58 \\
\hline$N=8$ & 12.3 & 2.5 & 23.26 & 23.16 & 0.38 & 0.39 \\
\hline$N=9$ & 24.9 & 1.6 & 25.72 & 25.64 & 0.24 & 0.23 \\
\hline$N=10$ & 48.2 & 1.0 & 28.18 & 28.13 & 0.12 & 0.12 \\
\hline$N=11$ & 48.2 & 14.0 & 30.65 & 30.61 & 0.03 & 0.03 \\
\hline$N=12$ & 77.2 & 6.1 & 33.11 & 33.09 & 0.05 & 0.05 \\
\hline$N=13$ & 13.6 & 1.2 & 35.63 & 35.58 & 0.11 & 0.23 \\
\hline$N=14$ & 10.5 & 0.3 & 38.10 & 38.10 & 0.27 & 0.27 \\
\hline$N=15$ & 9.1 & 0.08 & 40.57 & 40.58 & 0.30 & 0.30 \\
\hline
\end{tabular}



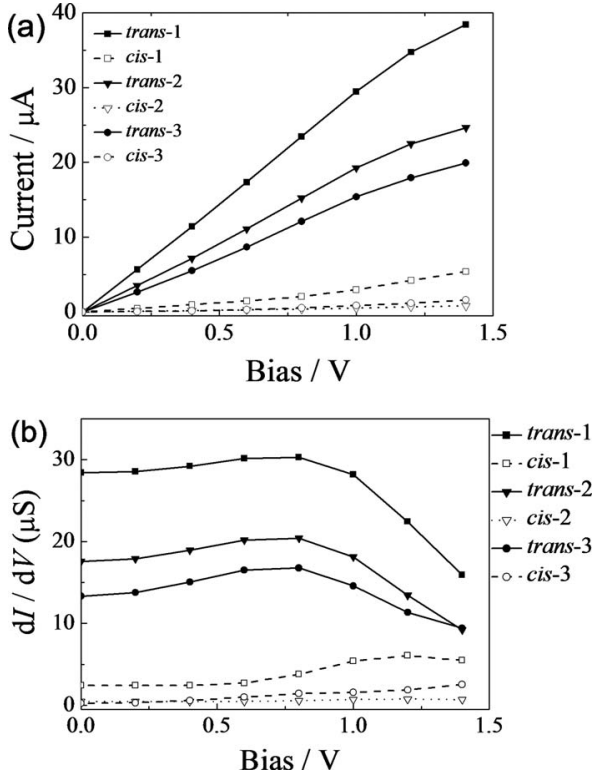

FIG. 3. (a) The $I-V$ curves and (b) the differential conductance of the trans $-N$ and $c i s-N$ series, typically for $N=1,2$, and 3. The solid and dashed lines represent the models of trans- $N$ and $c i s-N$, respectively.

$(28.5 \mu \mathrm{S})$ is about 12 times greater than that of model cis-1 $(2.4 \mu \mathrm{S})$. Experimental measurements also proved the large difference in conductance of naphthalene diamine. ${ }^{37} \mathrm{By}$ increasing the molecular length, the absolute value of conductance slightly varies, but the difference remains pronounced. Model trans $-N$ is more conductive than the corresponding cis- $N$, indicating that the electron transport is more efficient via the trans-linkage. This result also infers that different positions of terminal substitution may lead to various electron transport pathways.

Figure 3(a) illustrates the $I-V$ curves of the trans- $N$ and cis- $N$ molecular junctions. In the low bias region, all $I-V$ curves display a linear relationship before $1.0 \mathrm{~V}$. The differential conductance can be derived from the linearity, as shown in Fig. 3(b). In general, the differential conductance of the trans- $N$ series is always higher than $c i s-N$ regardless of chain length. The trans- $N$ series shows a slight variation before $1.0 \mathrm{~V}$ but decreases hereafter. On the contrary, cis- $\mathrm{N}$ series shows an increase when bias is higher than $1.0 \mathrm{~V}$. Although the high bias may reduce the conductance difference, it remains significantly even at $1.5 \mathrm{~V}$. The poor conductivity of the $c i s-N$ series also indicates the connection dependence of the electron transport behavior.

According to the Landauer formula, ${ }^{38}$ current is calculated by using the integration of the transmission coefficient in the bias window. To explore the origin of the terminal substitution effect, we further analyzed the transmission spectra. Figure 4 plots three typical transmission waves of trans- $N$ and $\operatorname{cis}-N(N=1,2$, and 3$)$ at zero bias. The first transmission peaks below and above the Fermi energy are mainly contributed by the HOMO and LUMO resonances, respectively. Since the HOMO energy level is closer to the Fermi level, the zero-bias conductance is most likely caused by the HOMO resonance wave. Comparing the trans- with cis-substitutions, we can find that the location of HOMO resonance is similar for each pair of models. This observa-

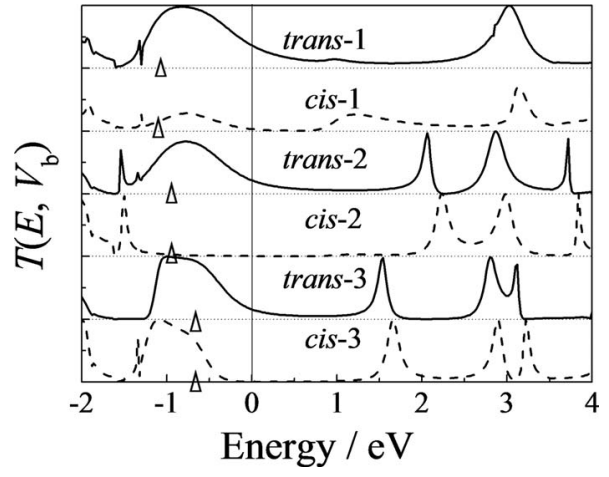

FIG. 4. The transmission $T\left(E, V_{b}\right)$ of trans- $N$ and $c i s-N$ models at zero bias, typically for $N=1,2$, and 3 . The up-triangles indicate the position of HOMO energy levels. The solid and dashed lines represent the models of trans- $N$ and $c i s-N$, respectively.

tion is consistent with other static features, such as energy levels and the spatial distributions. However, another two factors, e.g., the peak height and the wave attenuation, that reflect the dynamic transportation are different between the two models for each pair. From Fig. 4, we can find some of the trans-models exhibiting much stronger resonance wave than the cis-models do. For example, the peak height of model trans-1 (1.0) is much higher than that of cis-1 (0.28). On the other hand, the HOMO resonance declines faster toward the Fermi level $E_{f}=0$ for $\operatorname{cis}-N$ than $\operatorname{trans}-N$. For instance, the transmission coefficient at $E_{f}=0$ is 0.17 for trans-3, which is 70 times greater than that of cis-3 (0.0024), though they have the similar HOMO resonance position as indicated by the up-triangles. The substitution effect has also been demonstrated by others using various systems. Quinn $e t$ $a l^{39}$ investigated the conductance of naphthalene with diamino linkers. It was found that the 2,7-aminonaphthalene showed lower conductance than others due to the lack of aromatic stabilization. The theoretical prediction by Yoshizawa et al. ${ }^{40}$ was also in full agreement with our results, in which the electron transmission is forbidden in the 2,7-anthracene. Perrine et $a .^{41}$ also found the substitution effect in pentacene field-effect transistor.

The zero-bias transmission spectra are quite different between trans- $N$ and cis- $N$. Importantly, we also found that the difference is almost independent on bias. Therefore, the conductance difference between the two series is obvious even at bias as high as $1.4 \mathrm{~V}$. Figure 5 gives the third pair of models (anthracene) as an example to show the lack of bias dependence of transmission spectra. Here we can find that before $1.4 \mathrm{~V}$ both peak height and width of the transmission wave do not have obvious change. In particular, the LUMO peak shifts negatively from 1.56 to $1.44 \mathrm{eV}$ and the HOMO shifts a little from -0.68 to $-0.73 \mathrm{eV}$. Similarly, cis-3 also shows a very slight variation in these features. The lack of bias dependence of molecular projected self-consistent Hamiltonian is evidenced in Fig. 5(c). With respect to the energy levels at zero bias, all eigenstate values of trans-3 and cis-3 only slightly decrease, being responsible for the conductance difference.

To quickly summarize the above, trans- $N$ and cis- $N$ have the same molecular backbone but different terminal connec- 

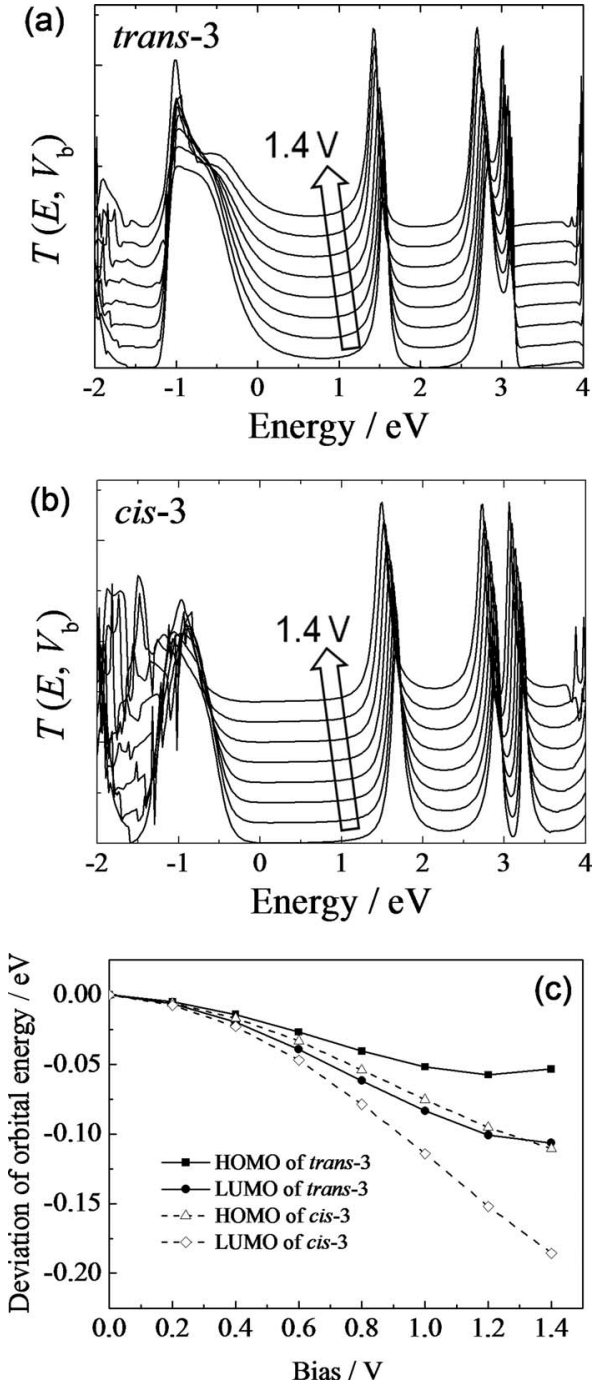

FIG. 5. The transmission $T\left(E, V_{b}\right)$ of (a) trans-3 and (b) cis-3 under various biases (shifted vertically by 0.1 for visibility). (c) The deviation of HOMO, LUMO relative to the zero bias of models $N=3$ with the bias.

tions. Due to this specific structural feature, the particular electron transport pathways is altered. The electron transport is efficient in the trans-connection, but impeded in the cisconnection.

\section{B. Intramolecular connection in the molecular bridge: The same electron transport pathway}

As discussed above, the molecular transportation strongly depends on the terminal connections. These results inspire us to further consider the influence of intramolecular connections. In order to reveal the role of intramolecular connection, we examined the BP- $N(N=1-8)$ series of models, which were structured on the basis of planar 4,4'-biphenyl dithiol. Compared to the polyacene molecular wires, this series of models has the same terminal connections to the electrodes but various linkages between the two benzene rings. Therefore, we may expect that more than one electron transport pathways exist. We first studied the static properties of these molecular junctions in detail.

Figure 6(a) plots the HOMO, LUMO energy levels, and HLG of the BP- $N$ series. The detailed values are listed in
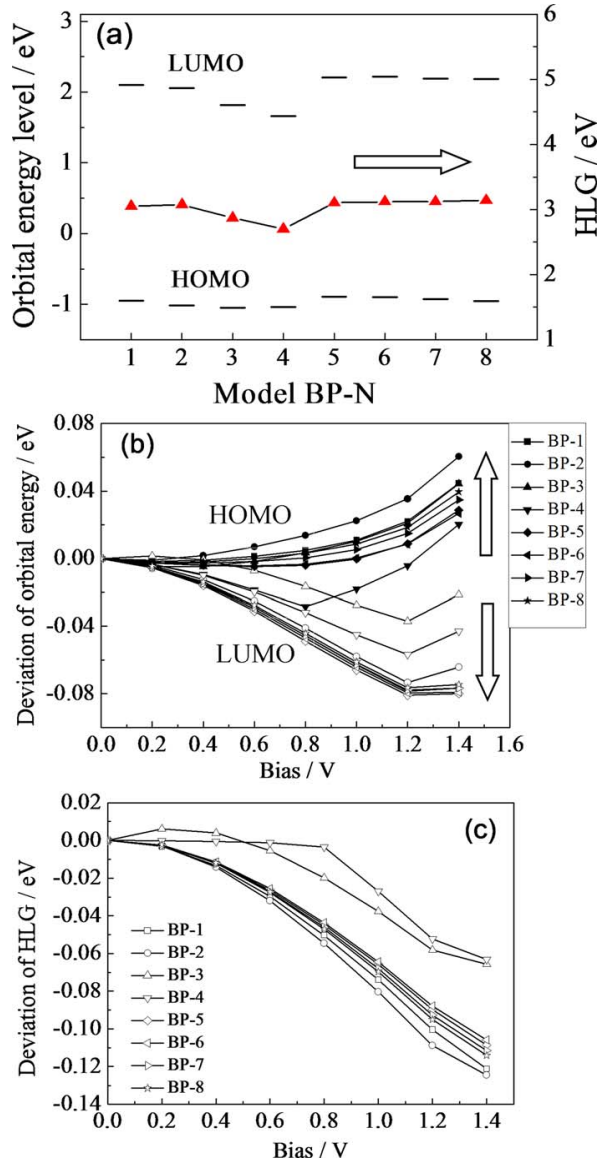

FIG. 6. (Color online) (a) The HOMO, LUMO energy level and HLG of model BP- $N(N=1-8)$ at zero bias. The short lines denote HOMO and LUMO and the triangles show the HLG. (b) The deviation of HOMO, LUMO (c) HLG relative to the zero bias of model BP- $N(N=1-8)$ with the bias. The solid and empty symbols show the HOMO and LUMO deviations, respectively.

Table II. The energy levels as well as the HLG decline from BP-1 to BP-4. BP-4 has the smallest HLG $(2.70 \mathrm{eV})$, because it has a larger $\pi$-overlap contributed by the two extra linkages. However, for the models from BP-5 to BP-8, the modification of extra linkage by $\mathrm{NH}, \mathrm{O}$, or $\mathrm{S}$ does not change the energy level of either HOMO or LUMO. The four molecules also have the similar HLG to BP-1 (4,4'-biphenyl dithiol). This result indicates that these extra linkages do not contribute to the frontier molecular orbitals for these models. We may also expect that they have similar transportation to BP-1. Another important difference among these models is the shift of HOMO and LUMO energy levels under the influence of bias [Fig. 6(b)]. Both the HOMO and LUMO slightly vary with the bias. The HOMO energy increases with the bias, whereas LUMO decreases. Consequently, HLG declines under the influence of bias [Fig. 6(c)].

The spatial distribution of the frontier molecular orbitals of the BP- $N$ series is presented in Fig. 7. Due to the participation of the extra conjugated bonds, model BP-4 displays different HOMO. The two extra linkages contribute much more to this orbital than the central $\mathrm{C}-\mathrm{C}$ bond does. On the contrary, only a limited part of the extra linkage contributes to HOMO in BP-2 and BP-3, or no contribution for other linkages from BP-5 to BP-8. However, the contribution of 
TABLE II. Molecular conductance, molecular length, HOMO, LUMO, and HLG of molecular junctions under zero bias of the BP- $N(N=1-8)$ system.

\begin{tabular}{|c|c|c|c|c|c|c|}
\hline No. & Name & $\mathrm{G} / \mu \mathrm{S}$ & Length/Å (S-M-S) & $\mathrm{HOMO} / \mathrm{eV}$ & LUMO/eV & $\mathrm{HLG} / \mathrm{eV}$ \\
\hline BP-1 & 4,4'-biphenyl dithiol & 10.8 & 10.92 & -0.95 & 2.10 & 3.05 \\
\hline BP-2 & 2,7-phenanthrene dithiol & 8.8 & 10.87 & -1.02 & 2.06 & 3.08 \\
\hline BP-3 & 9-N-2,7-phenanthrene dithiol & 8.4 & 10.85 & -1.05 & 1.82 & 2.87 \\
\hline BP-4 & $2,2^{\prime}$-pyrene dithiol & 9.9 & 10.81 & -1.04 & 1.66 & 2.70 \\
\hline BP-5 & 2,7-fluorene dithiol & 12.2 & 10.62 & -0.90 & 2.21 & 3.11 \\
\hline BP-6 & 2,7-carbazole dithiol & 12.5 & 10.54 & -0.90 & 2.22 & 3.12 \\
\hline BP-7 & 2,7-dibenzofuran dithiol & 11.9 & 10.49 & -0.93 & 2.19 & 3.12 \\
\hline BP-8 & 2,7-dibenzothiophene dithiol & 12.2 & 10.73 & -0.95 & 2.19 & 3.14 \\
\hline
\end{tabular}

extra linkages to LUMO varies. When only a single pathway is used in $\mathrm{BP}-1$, the central $\mathrm{C}-\mathrm{C}$ bond makes a large contribution to LUMO. When more linkages are added, the contribution from the central $\mathrm{C}-\mathrm{C}$ bond becomes less. From BP-5 to BP-8, we may observe that the main contribution comes from the central $\mathrm{C}-\mathrm{C}$ bond, but the contribution from the other linkage increases gradually. This diversity in LUMO promotes us to further study whether the transportation behavior has the essential difference among them.

Table II shows the conductance at zero bias and junction distance of BP- $N$ series. The conductance varies from 8.4 to $12.5 \mu \mathrm{S}$ for all BP- $N$ models. Although all of them have the similar molecular length (from 10.49 to $10.92 \AA$ ), the difference in conductance is almost negligible. The general trend of the $I-V$ curves of the molecular junctions is plotted in Fig. $8(\mathrm{a})$, in which all the $I-V$ curves show similar features. The current almost linearly increases with bias up to $0.8 \mathrm{~V}$. A nonlinear behavior is found in the larger bias region. The similarity of the transportation behavior for the BP- $N$ series is also observed in the differential conductance in Fig. 8(b). BP-4 does not show any significant difference from others, though it has different static properties, such as HOMO, LUMO, and spatial distributions. From these results we can conclude that the extra connections between the two benzene rings do not improve the electron transport anymore. Therefore, the central linkage $\mathrm{C}-\mathrm{C}$ bond is the main electron transport pathway in these molecular junctions.

In the transmission spectra (Fig. 9), the five representative models show similar transmission curves in the energy region between -1.0 and $1.0 \mathrm{eV}$. The waves centered at around $-0.8 \mathrm{eV}$ are derived from the HOMO states, which

\begin{tabular}{|c|c|c|c|c|c|}
\hline No. & Номо & LUMO & No. & номо & LUMO \\
\hline BP-1 & - 0000 & ogjogo & BP-5 & ంலిலீலి & - 88 \\
\hline BP-2 & - glứco & & BP-6 & - ชับำ & $-88^{\circ}$ \\
\hline BP-3 & 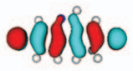 & . 8908. & BP-7 & o gిరిగిం & - \\
\hline BP-4 & $8 \% 8$. & . & BP-8 & ం రిலిరిం & 18:80 \\
\hline
\end{tabular}

FIG. 7. (Color) The HOMO and LUMO spatial distribution of BP-N $(N$ $=1-8)$ series at zero bias. make the main contribution to the molecular conductance. An important feature is the similar peak heights at $E_{f}=0$, which are in the range of $0.10-0.15$ and in good agreement with the similar conductance values listed in Table II.

By adjusting the intramolecular connections in conjugated molecules, we expect that more electron transport pathways exist. However, the similar conductance of the BP- $N$ series indicates that the electron may take the only one among the many pathways. This unique phenomenon could not be simply interpreted by the quantum interference effect. Therefore, an alternative explanation is required.

\section{The electron transport pathway}

In the macroscopic electric circuit, the electron favors to go through a wire with the lowest resistance. In a mesoscopic system such as protein (oxidase), the particular electron transport behavior is similar as demonstrated that electron goes through the low-resistance peptide chain. ${ }^{42}$ In a micro-
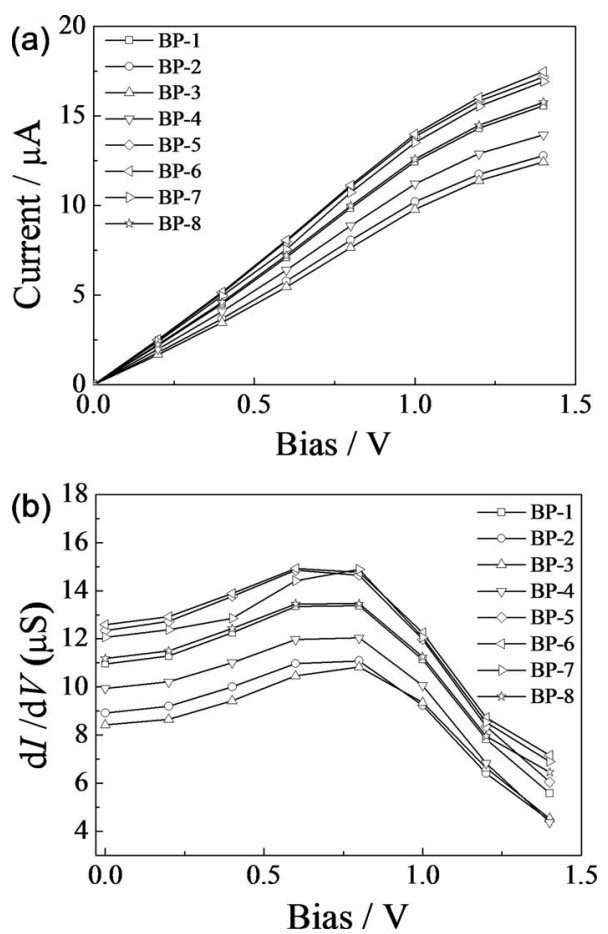

FIG. 8. (a) The $I-V$ curves and (b) the differential conductance of the model BP- $N(N=1-8)$ series. 


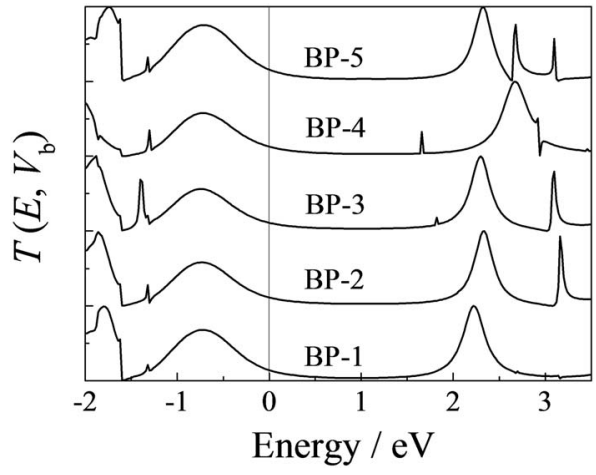

FIG. 9. The transmission spectra $T\left(E, V_{b}\right)$ of the model BP-N $(N=1-5)$ series at zero bias.

scopic porphyrin molecule, we also demonstrated that the electron transport pathway is the one with the lowest energy barrier. $^{20}$

One question raised is how to determine the electron transport pathway in present models. Efficient electron transport is dependent on the energy barrier, which is described by barrier height and barrier length. Compared to barrier length, barrier height is more important because most organic molecules follow an exponential decay of conductance. ${ }^{10}$ Our previous work has shown that the decay factor of all trans-polyacetylene is $0.036 \AA^{-1}$, which is much lower than others due to a narrow energy gap. Solomon et al. also confirmed that the long linearly conjugated molecule is more conductive than the short cross-conjugated one. ${ }^{43}$ They attributed this observation to the alternate singledouble bond structure in linearly conjugated molecule. From these facts, we can propose that the electron transport should be more efficient in those conjugated chains with alternate double and single bonds, such as polyacetylene. ${ }^{10,44}$ Thus the most probable electron transport pathway is the repeat units of double-single bonds, which has a lower energy barrier.

All the aromatic molecules can be represented by a number of different resonance structures. Figure 10 gives all possible resonance forms of anthracene for example. Trans-3 has five nonequivalent resonance forms and the resonance form $E$ is connected to $\mathrm{S}$ atoms through double bonds. Because two $\mathrm{S}$ atoms are positively charged with $0.12 e$, the resonance form $E$ should make more contribution to the conductance. The thick lines in Fig. 10 indicate the most probable electron transport pathway, which is -S-(double-single $)_{n}$-double-S- linkage. It is observed that at least one pathway is effective in trans-3. However, all four equivalent resonance forms of $c i s-3$ are inefficient to conductance. Because either the electron injecting or ejecting point is a single-single bond unit, as shown in thick lines. Therefore, the electron transport is impeded in such configuration. For the longer molecules, the number of resonance forms increases, thus, we expect that the difference between the two connections reduces. We should also mention that the quantum interference model proposed by Walter et al. ${ }^{17}$ is also available for interpreting the conductance difference between trans- and cis-substitution. According to their model, the constructive interference increases the conductance of trans-substituted naphthalene. On the contrary, the destruc-

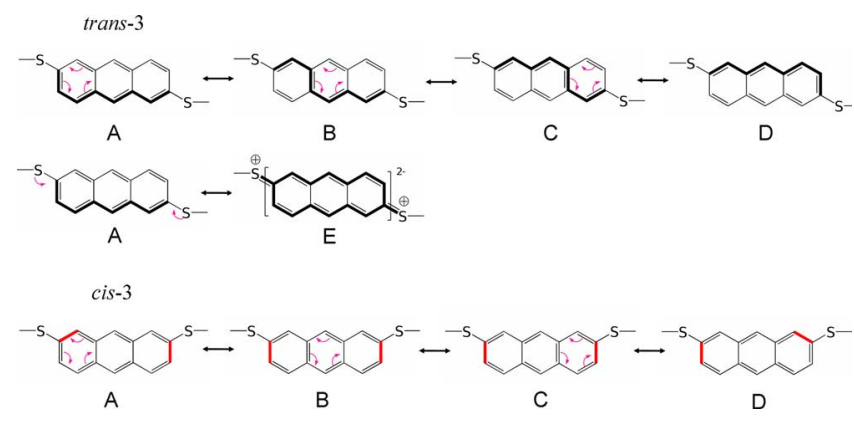

FIG. 10. (Color online) The resonance forms of trans-3 and cis-3, in which the thiol group loses the $\mathrm{H}$ atom when it attaches to the electrodes. The trans-3 can be represented by five resonance forms, while only four resonance forms contribute to $c i s-3$. The thick bonds denote the most probable electron transport pathway in trans-3 and the thick bonds impede electron transport in cis-3.

tive interference decreases the conductance of the cis-one. This model is especially proper to describe the electron transport in systems with two channels, such as [18] annulenes. $^{29,45}$

For the BP- $N$ series with different intramolecular connections, the substitution position of the $\mathrm{S}$ anchoring groups has been fixed. The electron injects from a $\mathrm{S}-\mathrm{C}$ bond and the following would be a double bond. It is clearly shown that, for all the models, the central -S-(double-single) ${ }_{3}$-double-Slinkage is the most probable one as indicated by thick lines in Scheme 2. However, the other pathways include singlesingle bonds that impede electron transport. In this case, extra connections between the two benzene rings do not modify the transport behavior. This result is in full agreement with the first series that the $-S$-(double-single $)_{n}$-double-S- linkage is the most probable pathway.

\section{CONCLUSIONS}

By using the DFT-NEGF method, we compared the transportation behaviors of two series of models with various terminal connections and intramolecular connections. The trans-like polyacene has different terminal connections from the cis-like one and, therefore, has different electron transport pathways. This has been evidenced by the unique transportation behaviors. On the other hand, the models BP- $N$ are composed of molecules with different intramolecular connections between the two benzene rings. Comparing with the terminal connection effect, the extra intramolecular linkages do not show obvious influence on the transportation behavior. These results confirm that the alternate double-single bond unit is the most probable electron transport pathway. The present study also indicates that not only the substitution effect should be considered in the design of molecular electronics, but also the particular linkage between the functional units.

\section{ACKNOWLEDGMENTS}

This work was supported by the National Natural Science Foundation of China (NSFC) (Grant Nos. 20821063 and 20873063) and the National Basic Research Program of China (973 Program No. 2007CB936302). 
${ }^{1}$ N. J. Tao, Nat. Nanotechnol. 1, 173 (2006); A. Nitzan and M. A. Ratner, Science 300, 1384 (2003).

${ }^{2}$ M. A. Reed, C. Zhou, C. J. Muller, T. P. Burgin, and J. M. Tour, Science 278, 252 (1997); R. H. M. Smit, Y. Noat, C. Untiedt, N. D. Lang, M. C. van Hemert, and J. M. van Ruitenbeek, Nature (London) 419, 906 (2002).

${ }^{3}$ B. Q. Xu and N. J. Tao, Science 301, 1221 (2003).

${ }^{4}$ D. J. Wold and C. D. Frisbie, J. Am. Chem. Soc. 122, 2970 (2000); J. W. Zhao and K. Uosaki, Nano Lett. 2, 137 (2002).

${ }^{5}$ J. G. Kushmerick, D. B. Holt, S. K. Pollack, M. A. Ratner, J. C. Yang, T. L. Schull, J. Naciri, M. H. Moore, and R. Shashidhar, J. Am. Chem. Soc. 124, 10654 (2002); J. G. Kushmerick, D. B. Holt, J. C. Yang, J. Naciri, M. H. Moore, and R. Shashidhar, Phys. Rev. Lett. 89, 086802 (2002).

${ }^{6}$ K. Slowinski, R. V. Chamberlain II, R. Bilewicz, and M. Majda, J. Am. Chem. Soc. 118, 4709 (1996); R. E. Holmlin, R. Haag, M. L. Chabinyc, R. F. Ismagilov, A. E. Cohen, A. Terfort, M. A. Rampi, and G. M. Whitesides, ibid. 123, 5075 (2001).

${ }^{7}$ W. Su, J. Jiang, W. Lu, and Y. Luo, Nano Lett. 6, 2091 (2006); M. Kula, J. Jiang, W. Lu, and Y. Luo, J. Chem. Phys. 125, 194703 (2006).

${ }^{8}$ J. Taylor, H. Guo, and J. Wang, Phys. Rev. B 63, 245407 (2001); M. Brandbyge, J.-L. Mozos, P. Ordejón, J. Taylor, and K. Stokbro, ibid. 65, 165401 (2002).

${ }^{9}$ S. K. Nielsen, M. Brandbyge, K. Hansen, K. Stokbro, J. M. van Ruitenbeek, and F. Besenbacher, Phys. Rev. Lett. 89, 066804 (2002).

${ }^{10}$ H. M. Liu, N. Wang, J. W. Zhao, Y. Guo, X. Yin, F. Y. C. Boey, and H. Zhang, ChemPhysChem 9, 1416 (2008).

${ }^{11}$ C. Li, I. Pobelov, T. Wandlowski, A. Bagrets, A. Arnold, and F. Evers, J. Am. Chem. Soc. 130, 318 (2008).

${ }^{12}$ A. Troisi and M. A. Ratner, J. Am. Chem. Soc. 124, 14528 (2002); Nano Lett. 4, 591 (2004).

${ }^{13}$ G. J. Ashwell, W. D. Tyrrell, and A. J. Whittam, J. Mater. Chem. 13, 2855 (2003)

${ }^{14}$ L. Venkataraman, Y. S. Park, A. C. Whalley, C. Nuckolls, M. S. Hybertsen, and M. L. Steigerwald, Nano Lett. 7, 502 (2007).

${ }^{15}$ M. Q. Long, L. L. Wang, K. Q. Chen, X. F. Li, B. S. Zou, and Z. Shuai, Phys. Lett. A 365, 489 (2007)

${ }^{16}$ F. Chen, X. L. Li, J. Hihath, Z. F. Huang, and N. J. Tao, J. Am. Chem. Soc. 128, 15874 (2006); L. Venkataraman, J. E. Klare, I. W. Tam, C. Nuckolls, M. S. Hybertsen, and M. L. Steigerwald, Nano Lett. 6, 458 (2006); B. Kim, J. M. Beebe, Y. Jun, X. Y. Zhu, and C. D. Frisbie, J. Am. Chem. Soc. 128, 4970 (2006).

${ }^{17}$ D. Walter, D. Neuhauser, and R. Baer, Chem. Phys. 299, 139 (2004)

${ }^{18}$ S. K. Maiti, Phys. Lett. A 366, 114 (2007); Org. Electron. 8, 575 (2007).

${ }^{19}$ Z. Xu, N. Li, X. Jin, Y. Li, H. Liu, and J. Zhao, Chem. Lett. 36, 1278 (2007)

${ }^{20}$ N. Wang, H. Liu, J. Zhao, Y. Cui, Z. Xu, Y. Ye, M. Kiguchi, and K. Murakoshi, J. Phys. Chem. C 113, 7416 (2009).
${ }^{21}$ Y. W. Li, J. W. Zhao, X. Yin, and G. P. Yin, ChemPhysChem 7, 2593 (2006).

${ }^{22}$ J. W. Zhao, Y. W. Li, H. M. Liu, P. Li, and G. P. Yin, J. Mol. Struct.: THEOCHEM 861, 7 (2008).

${ }^{23}$ O. D. Jurchescu, J. Baas, and T. T. M. Palstra, Appl. Phys. Lett. 84, 3061 (2004); A. Pezzella, L. Panzella, O. Crescenzi, A. Napolitano, S. Navaratman, R. Edge, E. J. Land, V. Barone, and M. d'Ischia, J. Am. Chem. Soc. 128, 15490 (2006).

${ }^{24}$ A. Dodabalapur, L. Torsi, and H. E. Katz, Science 268, 270 (1995).

${ }^{25}$ H. L. Cheng, W. Y. Chou, C. W. Kuo, Y. W. Wang, Y. S. Mai, F. C. Tang, and S. W. Chu, Adv. Funct. Mater. 18, 285 (2008).

${ }^{26}$ R. Baer and D. Neuhauser, Chem. Phys. 281, 353 (2002).

${ }^{27}$ R. Baer and D. Neuhauser, J. Am. Chem. Soc. 124, 4200 (2002).

${ }^{28}$ D. M. Cardamone, C. A. Stafford, and S. Mazumdar, Nano Lett. 6, 2422 (2006).

${ }^{29}$ S. H. Ke, W. T. Yang, and H. U. Baranger, Nano Lett. 8, 3257 (2008).

${ }^{30}$ M. J. Frisch, G. W. Trucks, H. B. Schlegel et al., GAUSSIAN 03, Wallingford CT, 2004

${ }^{31}$ X. Yin, H. Liu, and J. Zhao, J. Chem. Phys. 125, 094711 (2006).

${ }^{32}$ H. M. Liu, P. Li, J. W. Zhao, X. Yin, and H. L. Zhang, J. Chem. Phys. 129, 224704 (2008).

${ }^{33}$ H. Grönbeck, A. Curioni, and W. Andreoni, J. Am. Chem. Soc. 122, 3839 (2000)

${ }^{34}$ ATOMISTIX TOOLKIT, version 2.0, QuantumWise A/S, www. quantumwise. com

${ }^{35}$ C. C. Kaun, B. Larade, and H. Guo, Phys. Rev. B 67, 121411 (2003); C. C. Kaun and H. Guo, Nano Lett. 3, 1521 (2003).

${ }^{36}$ N. Troullier and J. L. Martins, Phys. Rev. B 43, 1993 (1991).

${ }^{37}$ J. R. Quinn, F. W. Foss, L. Venkataraman, and R. Breslow, J. Am. Chem. Soc. 129, 12376 (2007).

${ }^{38}$ R. Landauer, Phys. Lett. A 85, 91 (1981).

${ }^{39}$ J. R. Quinn, F. W. Foss, L. Venkataraman, M. S. Hybertsen, and R. Breslow, J. Am. Chem. Soc. 129, 6714 (2007).

${ }^{40}$ K. Yoshizawa, T. Tada, and A. Staykov, J. Am. Chem. Soc. 130, 9406 (2008).

${ }^{41}$ T. M. Perrine, R. G. Smith, C. Marsh, and B. D. Dunietz, J. Chem. Phys. 128, 154706 (2008).

${ }^{42}$ J. J. Regan, B. E. Ramirez, J. R. Winkler, H. B. Gray, and B. G. Malmström, J. Bioenerg. Biomembr. 30, 35 (1998).

${ }^{43}$ G. C. Solomon, D. Q. Andrews, T. Hansen, R. H. Goldsmith, M. R. Wasielewski, R. P. Van Duyne, and M. A. Ratner, J. Chem. Phys. 129, 054701 (2008).

${ }^{44}$ G. C. Solomon, D. Q. Andrews, R. R. Van Duyne, and M. A. Ratner, ChemPhysChem 10, 257 (2009).

${ }^{45}$ C. A. Stafford, D. M. Cardamone, and S. Mazumdar, Nanotechnology 18, 424014 (2007) 